\title{
Challenges of Mobile Devices' Resources and in Communication Channels and their Solutions
}

\author{
Rashid G. Alakbarov \\ Institute of Information Technology, Azerbaijan National Academy of Sciences, Azerbaijan \\ E-mail: t.direktor_muavini@iit.science.az
}

Received: 05 February 2020; Accepted: 30 March 2020; Published: 08 February 2021

\begin{abstract}
The article is dedicated to the development of cloudlet based mobile cloud computing (MCC) to address the restrictions that occur in the resources of mobile devices (energy consumption, computing and memory resources, etc.) and the delays occurring in communication channels. The architecture offered in the article more efficiently ensures the demand of mobile devices for computing and storage and removes the latency that occur in the network. At the same time, the tasks related to energy saving and eliminating delays in communication channels by solving the problems that require complex computing and memory resources in the cloudlets located nearby the user were outlined in the article.
\end{abstract}

Index Terms: MCC, mobile devices, communication channel, energy consumption, cloudlet.

\section{Introduction}

Today, mobile devices are irreplaceable in people's everyday lives. Applications installed on mobile devices require more resources. However, mobile devices still have limited resources. Users call, create or edit documents through mobile devices, use e-mail and social networks and make extensive use of software applications (handle images, online games, translation programs, multimedia programs, etc.) which demand high computing and memory resources. Limitations in the technical characteristics (battery life, lack of computing and memory resources, etc.) of mobile devices restricts the users to use multimedia software applications efficiently. Thus, the gap between mobile applications that require large computing and memory resources and the limited capabilities of mobile devices is widening. The rapid development of cloud technology helps to eliminate this gap.

It is known that capabilities of any mobile device (computing and memory resources) are limited. Users use them to solve problems that require large computing and memory resources. Cloud technologies are vastly used for these purposes. MCC are aimed to provide efficient and fast use of computing cloud services by the users. Worldwide rapid increase in use of mobile devices (notebooks, notepads, smartphones etc.) and their connection to computing clouds using relevant telecommunication technologies (GPS, 3G, 4G, Wi-Fi etc.) through internet, cause development of a new - MCC technology. MCC are a new paradigm created from integration of a wireless network (mobile network) and computing clouds, eliminating limitations of computing and memory resources on mobile devices and decreasing the energy consumed while solving the problem Mobile devices have become the main computing platform for many users. Recently developed applications require large computing and memory resources. The use of MCC, which reduces the resource constraints and energy consumption of mobile devices and network latency, is a topical issue $[1,2]$. When solving software applications on a mobile device, the processor and memory resources of the mobile device are fully involved in solving the mentioned tasks. The limited technical capacity of the mobile device does not provide fast solution of the task, which consumpts high energy and leads to its battery discharge. MCC is used to solve this problem. That is, the tasks to be solved are executed on remote cloud servers with higher technical capacities than the mobile devices, and the mobile device acts as a terminal. As a result, the mobile device saves its battery.

Currently, users benefit from centralized clouds computing that have high computing and memory resources. However, the data processed is not delivered to the users fast. Recent increase in the number of mobile device users of the MCC, remoteness of cloud servers, loading of the Internet network causes great delays in delivery of the processed information to the users, which causes problems in cloud computing [3]. If the user tasks are solved in the nearby servers, the delays and the energy consumption are reduced.

Thus, the development of cloudlet based MCC to address the restrictions that occur in the resources of mobile devices (energy consumption, computing and memory resources, etc.), as well as the delays occurring in communication channels is of great importance. 


\section{Related Work}

For overcoming the lack of resources of cloudlet networks, the issue of optimal distribution of user applications among cloudlets is explored [4]. Depending on the type of software used by users, to reduce the energy consumption and delays, the question of offloading to the nearest clouds is considered [5]. The issues of saving energy on mobile devices using cloudlets and reducing network latency are considered [6, 7]. Delays in the use of remote cloud servers over the Internet (WAN) are analyzed and it is suggested to use cloudlet networks to eliminate them [8]. Article [9] researches cloudlet access capabilities of the users, duration of connection between the cloudlet and the user, issue solution time. The article [10] stresses that security issues are highly addressed when using cloudlet-based networks rather than when using cloud servers. The cloud servers located close to users greatly diminishes the latency in data sharing [11].

Some studies [12] are dedicated to the issues of development of a cloudlet network infrastructure located near Access Points with many Wireless Metropolitan Area Networks (WMAN) users. Therefore, it is proposed to place the cloudlets near venues with higher number of mobile users (malls, libraries, schools, universities, stadiums, stations, airports etc.). Article [13] reviews issues of issues of data cashing on cloudlets in order to reduce delays. The article [14] discusses the issue of eliminating delays and reducing the cost of services through the optimal distribution of mobile users' tasks on cloud.

In some research works, they tried to minimize energy consumption and latency in communication channels through properly locating main and auxiliary parts of software applications that are utilized by the users in mobile and cloud servers [15]. Article [16] develops a method for providing effective usage of cloud resources by the mobile users. The paper offers solution for correct use of cloudlets located along the movement route of the mobile users in (WMAN) environment. Article [17] describes cloudlets placed near to the users provide faster processing of user data in comparison with further located cloud servers and reduce energy consumption. Such kind of connection ensures less network loading, and processed data is received faster. Some research [18] explore some problems related to planning of geographically distributed network, optimal location of cloudlets close to base stations and optimization of network parameters. The article [19] shows how to save energy consumption by ensuring a balanced distribution of user issues in cloudlets.

\section{Problems Occurring in the Mobile Devices}

This section describes a framework for automatic cloud service monitoring and management. It also provides an approach introduced using this framework. Services rendered over internet require infrastructures with high sustainability and bandwidth capabilities. Regardless of the type of architecture used in development of the MCC, created infrastructure must be reliable. Bandwidth of the created network must be high in order to eliminate the delays. Factors that affect processing and delayed data delivery to the users on MCC are following [20, 21].

- information processing period on cloud servers;

- delays occurring in network loading;

- bandwidth diversity of the communication channel that provides data transfer.

Numerous actions are performed to address the latency in network. Closer the application is located to the users, the more minimized is the delay problem on the network. Users when solving the task on remote servers must take into account that, delays on the communication channel hinder efficient solution of complex issues that require large computing and memory resources on the mobile device. In order to eliminate the limitations of the computing resources, centralized MCC is used, created from integration of mobile network and computing clouds. Despite continuous improvement of the mobile devices, the resource limitation difference between mobile devices and other non-mobile devices will always persist [6].

The problems related to the effective usage of MCC may include:

- cloud servers of cloud computing systems located far away from the mobile users;

- long delivery of data and results to the users;

- lack of resources of mobile devices;

- breakages occurring in the communication channels;

- limited battery life in mobile devices;

- security issues of communication channels;

- inappropriate organization of data migration among cloudlets;

- usage of cloudlets with various technical capacities in the network; 
- non-optimal deployment of user tasks in cloudlets;

- etc.

The article provides solutions for some abovementioned problems (increasing battery life of mobile devices, lack of resources of mobile devices, fast delivery of data and results to users) with the use of cloudlet-based mobile computing clouds.

Limited performance period of the batteries is one of the main problems of the mobile devices. Implementation of the application of the clouds reduces energy consumption on the mobile device. However, it is impossible to completely transfer implementation of all applications to the cloud. For example, starting the application, data input and on-screen demonstration of processing results must certainly be performed on the device. Only implementation of the application can be done on the cloud server. Energy of the mobile devices is used to display different information on the screen, solution of certain part of the problems and internet connection [22]. Use of social networks, online applications, web pages and telephone calls causes emptying of the battery and requires its frequent connection to the power outlet. Current technologies cannot ensure long-term operation of the batteries of the mobile devices. Cloud technologies are introduced to prolong performance period the batteries (energy saving). If software applications that require large computing and storage resources are used, processor of the mobile device and memory resources participate in problem solution with full force. This causes fast emptying of the battery. Cloud technology is used in order to eliminate this problem, i.e. solved problem in directed to the cloud server and mobile device acts as a terminal in this case. Consequently, it ensures long term performance of its battery. At the same time, mobile devices $23 \%$ less energy while using Wi-Fi in comparison with other connection technologies. Also, closer the software application to the mobile devices, faster the problems are solved due to reduction of delays on the communication channel and thus, less the energy requirement of the mobile device. Scarcity of resources must be taken into consideration while reviewing mobile devices in the cloud environment. Although multiple parameters such as processor and memory resources, screen size, wireless communication, sensor technology and operations are significantly improved, there are still serious problems in use of energy source and exploitation of complex applications. By deficiency of resources of the mobile devices, we mean limitations in their computing and memory resources. Technical capabilities of the smartphones that use operation systems such as Android and Windows Mobile falls behind personal computers: 3 times in computing capacity, 8 times in memory, 10 times network bandwidth [23]. Overall, it must be noted that, resource deficiency is one of the main reasons behind development of MCC. In order to eliminate this deficiency of the mobile devices required resources are added to cloud infrastructure and used by the user at any time. By providing users with the solution of queries in nearby cloudlets, it is possible to eliminate delays and to ensure reliable operation of the network by reducing the number of communication channels between users and cloudlets.

The smaller the number of nodes between the cloudlet and the user, the less the delays. This, in turn, helps to deliver the results and data to the user faster. The number of communication channels between users and the cloudlet should be minimal, which ensures reliable operation of communication channels.

\section{Cloudlet based Mobile Cloud Computing Architecture}

Cloudlets are developed close to base stations of the mobile operators to benefit from cloud technologies by mobile equipment. Cloudlet - is a computer connected to the Internet. Its computing capability is less than that of cloud servers but higher than that of mobile devices [24]. Cloudlets eliminate delays in transfer of data to the mobile device from remotely located cloud servers located.

Cloudlets should be located close to mobile device to minimize the latency. Ideal place for locating cloudlets is the nearest base stations to the mobile device [25]. For instance, we can place cloudlet at the mobile base station (3G/4G) or the access point (Wi-Fi). Services needed by the users are rendered through cloudlets. If a user must work with a certain software application, he calls that application to the cloudlet near him, uses it for a necessary period of time and sends that application back to the central server. It minimizes clouds usage service fee, task solution time and lightly loads the network.

Fast service access, mobility support, and minimized roaming fees, are the main benefits of cloudlets. Since software applications of users are placed next to cloudlets, data can be accessed by users immediately, eliminating network latency. Correspondingly, as in cloud computing, the technical capacities of cloudlets become higher than the those of mobile devices. This ensures software applications, which are not handled on a mobile device, run in cloudlets. Hierarchically structured cloudlet-based mobile computing systems are beneficial for such kind of task solution.

The architecture of cloudlet-based MCC is presented in Fig.1. 


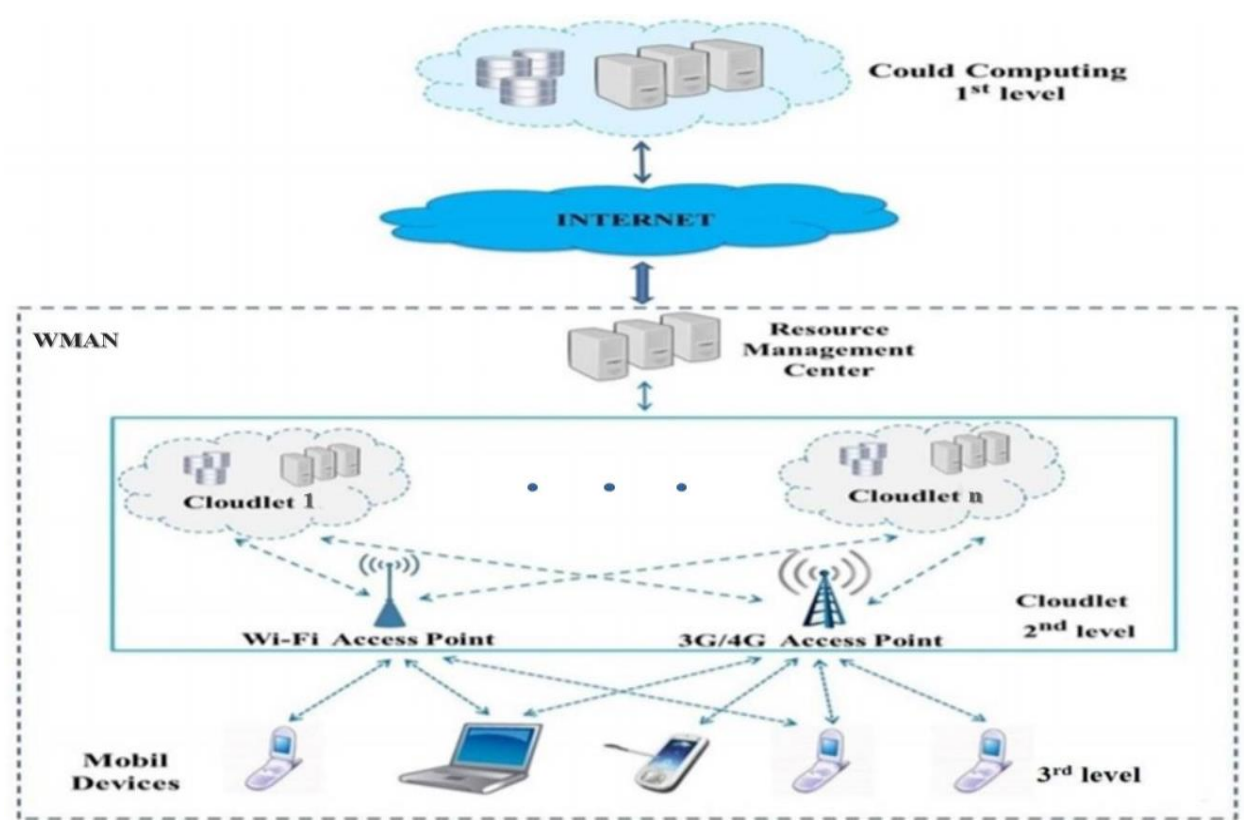

Fig.1. Architecture of cloudlet based mobile cloud computing

The hierarchically structured MCC consists of three layers. Layer 1 contains the servers of the cloud computing system, Layer 2 contains the cloudlets near the base stations, and Layer 3 contains mobile devices. Thus, a cloudlet is established next to the base stations of mobile operators for the extensive and more efficient use of cloud technologies in mobile equipment. Cloudlets are managed by the Resource Management Center (RMC) developed in the second layer $[16,20]$. The cloud services requested by users are employed via cloudlets, and this increases the service quality. When mobile users access a cloudlet network, they first connect to the RMS, which then connects the user to the appropriate cloud servers remotely over the Internet for a while. Requests and data from mobile users are transmitted to the RMS, which provides services to the mobile network. RMS gathers data about the technical capabilities of cloudlets and the location of mobile users close to the cloud. Therefore, based on the request received from a mobile user, RMS provides the deployment of the application requested from the cloud servers in the cloudlet that satisfies the demand of user. Mobile users offload the applications to the nearest cloudlets and process them, which in turn avoids the Internet overload. This architecture, albeit partially, increases the performance of some of the above options (task solution time, latency, breaks, etc.). Advantages of using cloudlets may be fast access service, mobility support, reduction of roaming fees. Thus, solving the user applications in nearby cloudlets eliminates the resource shortages on mobile devices, network delays, and minimizes the power consumption of the mobile device. Correspondingly, the technical capabilities of cloudlets become higher than the those of mobile devices, and this allows faster processing of long-term software applications on mobile devices in cloudlets. Alternatively, security issues (minimizing DoS attacks on mobile devices) in hierarchically structured mobile computing clouds and are more reliably provided in communication channels because software tools are located in cloudlets next to the mobile user. Since centralized mobile computing clouds connect the mobile user and the remote cloud server is via the Internet, the power of unsanctioned intrusions into the communication channels increases and security support becomes more problematic. Let's view the analysis of energy consumption and network latency when using cloudlets or cloud servers located remotely from the mobile user.

\section{Problem Solution}

Let's use following markings:

computing productivity of mobile device and cloudlet (number of commands performed during a unit of time) $M$ and $S$;

solution periods of issue in mobile device and cloudlet $-T_{m}$ and $T_{s}$;

number of instructions (commands) in the issues to be solved - $C$;

data transfer speed on the communication channel (between mobile device and cloudlet) - $B$ (bit/sec); volume of data transferred and accepted between the cloudlet and the mobile device - $D$ (bite);

energy used by the mobile device and cloudlet for solution of a problem (during a unit of time) $-P_{1}^{m}$ and $P_{1}^{s}$; energy consumed by the mobile device for data transfer and obtaining results (during a unit of time) - $P_{2}^{m}$; data transfer speed to communication channels - $V$;

average data transfer speed on communication channels $-V_{\text {average }}$. 
For example, solution time on a mobile device and cloudlet will be $T_{m}=\frac{C}{M}$ and $T_{S}=\frac{C}{S}$. Time spent on data transfer and accepting results between the cloud and the mobile device will be $T_{d}=\frac{D}{B}$. Energy used by the mobile device to solve a problem will be $E_{m}=P_{1}^{m} * \frac{C}{M}$.

Energy spend while solving and delivering the problem to the use will be

$$
E_{s}=P_{1}^{s} * T_{s}+P_{2}^{m} * T_{d}=P_{1}^{s} * \frac{C}{S}+P_{2}^{m} * \frac{D}{B}
$$

Gain obtained in energy consumption while the mobile device solves the problem on the cloudlet is calculated as following:

$$
E=E_{m}-E_{s}=P_{1}^{m} * \frac{C}{M}-P_{1}^{s} * \frac{C}{S}-P_{2}^{m} * \frac{D}{B}
$$

Let's assume that, given the computing power $(S)$ of the computers used in the cloud is $N$ (let's assume=3) times higher than the computing power $(M)$ of the mobile device $(S=3 M)$ then (1) formula will be as following:

$$
\begin{aligned}
& E=P_{1}^{m} * \frac{C}{M}-P_{1}^{s} * \frac{C}{N M}-P_{2}^{m} * \frac{D}{B}=P_{1}^{m} * \frac{C}{M}- \\
& P_{1}^{s} * \frac{C}{3 M}-P_{2}^{m} * \frac{D}{B}=\frac{C}{M} *\left(P_{1}^{m}-P_{1}^{s} * \frac{C}{3}\right)-P_{2}^{m} * \frac{D}{B}
\end{aligned}
$$

If the value of formula (2) is a positive number, then mobile device will consume less energy as much as that number. As seen from formula (2), $D / B$ number must be less, and $C / M$ and $N$ numbers must be higher in order for the number $E$ to be positive. In this case, mobile device can consume less energy by sending the problem to the cloudlet and solving it there.

It's more appropriate to use cloudlets to solve problem that require large-scale computing and memory resources and small-sized data exchange (transfer and receipt) issues.

Multifold greatness of computing power of computers used at the cloudlets over mobile devices will ensure raid solution of the problem. On the other hand, mobile device only participates in data transfer during problem solution and obtaining results. This caused low energy usage. In its turn, this reduced energy consumption of the mobile device and facilitates long-term operation of the battery.

Eliminating the delays in communication channels, we can reduce consumption of energy in mobile devices. Mobile devices use multiple communication channels (wireless mobile and internet network) while connecting to the server using 3G/4G technology. Multitude and diversity of communication channels (use of channels with different bandwidths, disconnections, overloading Internet network etc.) affects data transfer speed. If data transfer speed is low, mobile device will use more energy to transfer data. Mobile users use nearby cloudlets in order to increase transfer speed, which in its turn, increases data transfer speed.

$n$ number of intermediary communication channels is used to transfer data $(D)$ when a problem is solved in remote computing clouds. If transfer speed of each intermediary channels is marked as $V_{j}=\frac{D}{t_{j}}$, then final average speed will be

$$
V_{\text {average }}=\frac{v_{1} t_{1}+v_{2} t_{2}+\ldots+v_{n} t_{n}}{t_{1}+t_{2}+\ldots+t_{n}}=\frac{\sum_{k-1}^{n} v_{k} t_{k}}{\sum_{k-1}^{n} t_{k}}
$$

Time spent to transfer data to the remote cloud server and obtain the result will be

$$
T_{\text {average }}=\frac{D}{V_{\text {average }}}=D * \frac{\sum_{k-1}^{n} t_{k}}{\sum_{k-1}^{n} v_{k} t_{k}}
$$

This time $\left(T_{\text {average }}\right)$ will be higher than transfer time $\left(T_{d}=\frac{D}{B}\right)$ of the direct communication channel $\left(T_{\text {average }}>T_{d}\right)$. In its turn, solution of the problem on the cloudlet causes low energy consumption by the mobile device. For this reason, 
we should try to have a few communication channels between the mobile device and the cloudlet, and use Wi-Fi for connection.

Article experimentally compares energy consumption while downloading files from the cloudlet and remote cloud server to the mobile device and delays occurring in communication channels.

\section{Comparison and Evaluation of Experimental Results}

Let's compare the results of the experiments conducted suing cloud server and the cloudlet remotely located from mobile users in the MCC. The mobile user uses $4 \mathrm{G}$ to connect to cloud, while he/she uses (Wi-Fi) to join the cloudlet.

In this time, we developed two dissimilar scenarios for mobile computing to assess the offered model. The first scenario connects the mobile telephone to the corporate remote cloud server using $4 \mathrm{G}$. Whereas, the second scenario connects the mobile device to the cloudlet (CL) using Wi-Fi. Cloudlet is connected to the remote cloud server using cables. Noted both connection experiments were actually verified. Following equipment was used for performed experiments:

Cloudlet server: Lenovo V110 laptop (Core i5- 6200, CPU- 2.4 Ghz, RAM - 4 Gb, HDD (SSD) - 256 gb)

Access device: Cisco - WS2950 PO-8TT-L router connected to Internet

Mobile phone: Samsung Galaxy Note 8 Exynos Octa 8895 (CPU - 2.3 Ghz, RAM 6 Gb, HDD (SSD) - 64 gb.

Experimental tests include offloading 4 (four) files (of 10, 40, 66, 108 Mbytes) from a mobile device to a cloudlet or a remote cloud server. Power consumption and delays when offloading files to a mobile device are compared.

Expenses during both scenarios were calculated. Table 1 shows the outcomes attained from the Cloudlet (Wi-Fi) and remote institutional cloud (4G). Expenses were calculated considering mobile internet costs ( $1 \mathrm{~Gb}-5 \$)$.

Table 1. Comparison of cloud server and cloudlet

\begin{tabular}{|c|c|c|c|c|c|}
\hline \multirow{2}{*}{ File Size } & Connection & $\begin{array}{c}\text { Energy consumption } \\
\mathbf{m W}\end{array}$ & $\begin{array}{c}\text { Delays in } \\
\text { seconds }\end{array}$ & $\begin{array}{c}\text { Transfer } \\
\text { speed } \\
\text { Mbit/sec }\end{array}$ & Expenses (\$) \\
\hline \multirow{2}{*}{$10 \mathrm{Mb}$} & Cloudlet - Wi-Fi & 31,3 & 0,49 & 20,1 & $\approx 0$ \\
\cline { 2 - 5 } & Cloud Server-4G & 59,4 & 1,16 & 8,6 & 0,05 \\
\hline \multirow{2}{*}{$40 \mathrm{Mb}$} & Cloudlet - Wi-Fi & 42,7 & 1,96 & 20,4 & $\approx 0$ \\
\cline { 2 - 6 } & Cloud Server-4G & 78,3 & 3,57 & 11,2 & 0,2 \\
\hline \multirow{2}{*}{$66 \mathrm{Mb}$} & Cloudlet - Wi-Fi & 72,6 & 3,17 & 20,8 & $\approx 0$ \\
\cline { 2 - 6 } & Cloud Server-4G & 82,6 & 4,58 & 14,4 & 0,33 \\
\hline \multirow{2}{*}{$108 \mathrm{Mb}$} & Cloudlet -Wi-Fi & 82,8 & 5,02 & 21.5 & $\approx 0$ \\
\cline { 2 - 6 } & Cloud Server-4G & 98,1 & 6,54 & 16,5 & 0,54 \\
\hline
\end{tabular}

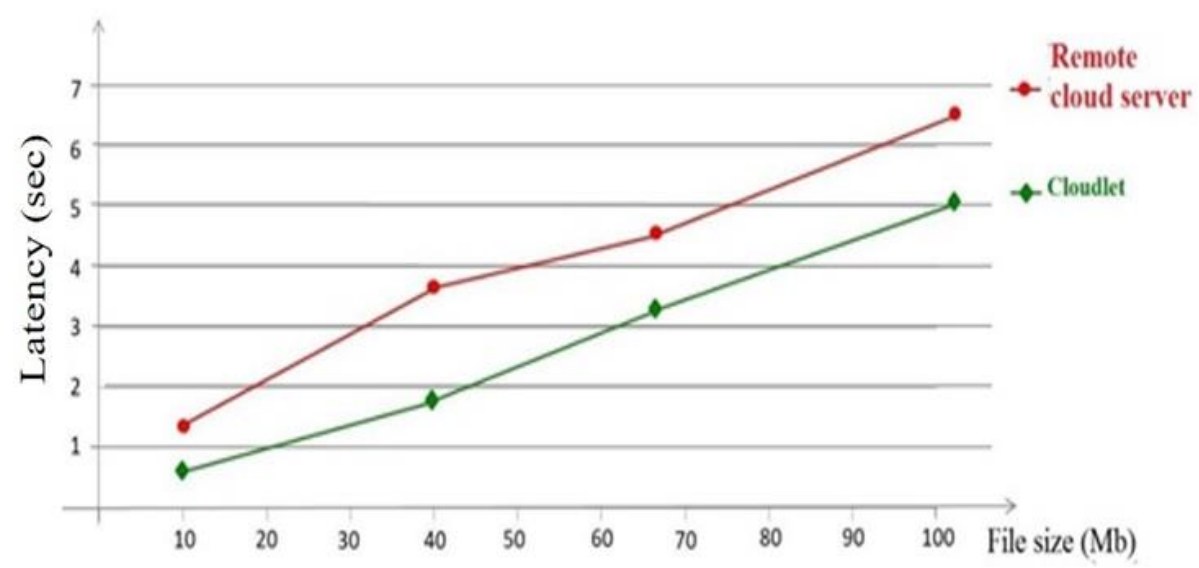

Fig.2. Comparison of delays in file loading from cloud server and cloudlet 


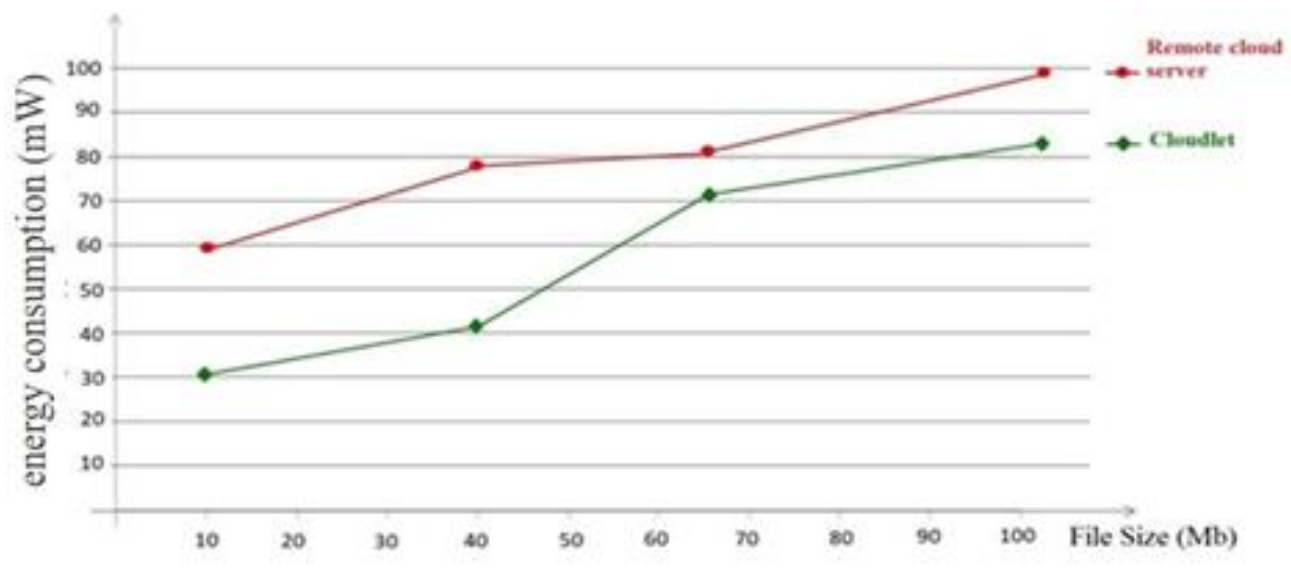

Fig.3. Comparison of energy consumption of the mobile device

Fig.3 Depicts the diagram of comparative analysis of energy consumption of the mobile devices while using both scenarios.

Performed experiments show the advantage of the cloudlet over remote cloud server. Thus, when the mobile device downloads a $100 \mathrm{Mb}$ file from the cloudlet, it consumer 1.2 times less energy in comparison with its download from a remote cloud server. At the same time, transfer speed of the channel is 1.3 times higher. When remote cloud servers are used, transfer of large files requires a lot of time and delays occurring during use of clouds can be considered acceptable. Therefore, it has been confirmed that cloudlet-based mobile clouds are more efficient in reducing the device's energy consumption and in rapid processing of software applications implemented in the cloudlet rather than the clouds located far from the user.

\section{Future Research Directions}

In future research, we are going to design methods and algorithms to resolve the issues, such as monitoring of deployment (near educational institutions, shopping malls, leisure areas, etc.) of cloudlets in MCC, the prediction of location of cloudlets close to base stations, the development of methods and algorithms for choosing the right virtual machines to reduce task solution costs in cloudlets, identification of high-intensity software applications and their clustering in cloudlets, optimal placement of mobile applications in cloudlets located on the route and their effective and well-adjusted use in the cloudlet resources, placement of user software in the cloudlet connected to the user with minimal communication channels, etc.

\section{Conclusions}

The Article consider that proposed cloudlet based hierarchically structured mobile computer cloud ensures the eradication of shortages in energy consumption, computing and storage resources in the mobile devices. Problems occurring in MCC and their solution methods were analyzed. When solving the tasks in the cloudlets the low energy consumption of mobile devices is shown with mathematical computation which provides long battery life. As a result of the experiment, it was confirmed that the use of cloudlets is more efficient. Thus, when downloading a 100 MB file from the cloudlet through a mobile device, the energy consumption is 1.2 times less than downloading from a remote cloud server, and at the same time, the transmission rate of the channel is 1.3 times higher. At the same time, reducing solution time for problems, reduced network loading, elimination of delays and increase of reliability by the cloudletbased network was demonstrated.

\section{References}

[1] G. Lu, W. H. Zeng, "Cloud computing survey”, Applied Mechanics and Materials, vol. 530, pp. 650-661, 2014.

[2] R. Buyya, A. Beloglazov, J. Abawajy, "Energy-efficient management of data center resources for cloud computing: a vision, architectural elements, and open challenges", Proceedings of the 2010 International Conference on Parallel and Distributed Processing Techniques and Applications, 2010, pp. 1-12.

[3] S. Abolfazli, "Cloud-based Augmentation for Mobile Devices: Motivation, Taxonomies, and Open Challenges", IEEE Communications Surveys \& Tutorials, vol. 16, no.1, pp. 337-368, 2014.

[4] M. Z. Nayyer, I. Raza, S. A. Hussain, "A Survey of Cloudlet-Based Mobile Augmentation Approaches for Resource Optimization”, ACM Computing Surveys, vol. 51, no. 5, pp.107-135, 2018.

[5] D. G. Roy, D. De, A. Mukherjee, R. Buyya, "Application-aware cloudlet selection for computation offloading in multi-cloudlet environment”, Journal of Supercomputing, vol. 73, pp. 1672-1690, 2017. 
[6] A. Mukherjee, P. Gupta, D. De, "Mobile cloud computing based energy efficient offloading strategies for femtocell network", Proceedings of the International Conference on Applications and innovations in mobile computing, IEEE, 2014, pp. $28-35$.

[7] E. Ahmed, A Gani, M.K Khan, R. Buyya, S.U Khan, "Seamless application execution in mobile cloud computing: motivation, taxonomy, and open challenges", Journal of Network and Computer Applications, vol. 52, pp. 154-172, 2015.

[8] A. Beloglazov, J. Abawajy, R. Buyya, "Energy-aware resource allocation heuristics for efficient management of data centers for cloud computing", Future Generation Computer Systems, vol. 28, issue 5, pp.755-768, 2012.

[9] M. Jia, W. Liang, Z. Xu, M. Huang, "Cloudlet load balancing in wireless metropolitan area networks", The 35th Annual IEEE International Conference on Computer Communications, 10-14 April, 2016, pp. 730-738.

[10] M. Quwaider, Y. Jararweh, "Cloudlet-based efficient data collection in wireless body area networks", Simulation Modelling Practice and Theory, vol. 50, pp. 57-71, 2015.

[11] Y. Li, W. Wang, "The unheralded power of cloudlet computing in the vicinity of mobile devices", Global Communications Conference (GLOBECOM), 2013 IEEE, pp. 4994-4999.

[12] Y. Jararweh, Z. Alshara, M. Jarrah, M. Kharbutli, M.N. Alsaleh, "TeachCloud: a cloud computing educational toolkit", International Journal of Cloud Computing, 2013, 2, (2/3), pp. 237-257.

[13] Z. Pang, L. Sun, Z. Wang, E. Tian, and S. Yang, "A Survey of Cloudlet based Mobile Computing", 2015 International Conference on Cloud Computing and Big Data, 2015, pp. 268-275.

[14] A. Aghdashi, S. L. Mirtaheri, "A Survey on Load Balancing in Cloud Systems for Big Data Applications", International Congress on High-Performance Computing and Big Data Analysis, 2019, pp. 156-173.

[15] Huerta-Canepa., D. Lee, “A virtual cloud computing provider for mobile devices”, International Journal of Advance Research, Ideas and Innovations in Technology, vol. 3, no.3, 2017, pp. 411-414.

[16] R.K. Alekberov, O.R Alekperov, "Procedure of effective use of cloudlets in wireless metropolitan area network environment", International Journal of Computer Networks \& Communications (IJCNC), vol. 11, no.1, 2019, pp. 93-107.

[17] T. Diaby, B.B. Rad, "Cloud Computing: A review of the Concepts and Deployment Models", International Journal of Information Technology and Computer Science, vol. 9, no.6, 2017, pp. 50-58.

[18] M. Mam, G. Leena, N. S. Saxena, "Improved K-means Clustering based Distribution Planning on a Geographical Network", International Journal of Intelligent Systems and Applications, vol. 9, no.4, 2017, pp. 69-75.

[19] M. İ. Bala, M.A. Chishti, "Load Balancing in Cloud Computing Using Hungarian Algorithm", International Journal of Wireless and Microwave Technologies, vol. 9, no.6, 2019, pp. 1-10.

[20] E. Gelenbe, R. Lent, M. Douratsos, "Choosing a local or remote cloud", Proceedings of 2nd International Symposium on Network Cloud Computing and Applications, 2012, pp. 25-30.

[21] P. Gupta, S. Gupta, "Mobile Cloud Computing: The Future of Cloud", 'International Journal of Advanced Research in Electrical', Electronics and Instrumentation Engineering, vol. 1, no.3, 2012, pp.134-144.

[22] M. Shiraz, A. Gani, "Mobile Cloud Computing: Critical Analysis of Application Deployment in Virtual Machines", International Proceedings of Computer Science \& Information Tech, 2012, 27, pp. 11-16.

[23] D. Sarddar, R. Bose, “A Mobile Cloud Computing Architecture with Easy Resource Sharing”, International Journal of Current Engineering and Technology, vol. 4, no.3, 2014, pp. 1249-1254.

[24] N. Fernando, S.W. Loke, W. Rahayu, "Mobile cloud computing: A survey', Future Generation Computer Systems", vol. 29, 2013, pp. 84-106.

[25] M. Chen, Y. Hao, Y. Li, C.F. Lai, D. Wu, "On the computation offloading at ad hoc cloudlet: Architecture and service modes", IEEE Communications Magazine, vol. 53, 2015, pp. 18-24.

\section{Authors' Profiles}

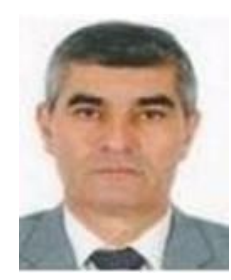

Rashid Alakbarov. Deputy director on technology of Institute of Information Technology of Azerbaijan National Academy of Sciences, Azerbaijan, Baku,

Born on February 2, 1953. PhD (2006), Assoc. Prof. (2016).

Currently, he conducts researches on the optimal allocation of memory and computing resources among users based on cloud technologies in Data processing center, on development of models for the synthesis of cloud technologies in various network environment and new methods and algorithms for the synthesis of e-science and its functional subsystems. Total number of research publications are 105.

How to cite this paper: Rashid G. Alakbarov, "Challenges of Mobile Devices' Resources and in Communication Channels and their Solutions", International Journal of Computer Network and Information Security(IJCNIS), Vol.13, No.1, pp.39-46, 2021. DOI: 10.5815/ijcnis.2021.01.04 